\title{
Grazing Incidence Resonant Soft X-ray Scattering for Analysis of Multi- Component Polymer-Fullerene Blend Thin Films
}

Christoph J. Schaffer, Cheng Wang ${ }^{2}$, Alexander Hexemer², and Peter MüllerBuschbaum

Technische Universität München, Physik-Department, Lehrstuhl für Funktionelle Materialien, James-Franck-Str. 1, 85748 Garching, Germany

${ }^{2}$ Advanced Light Source, Lawrence Berkeley National Laboratory, 1 Cyclotron Road, Berkeley, California 94720, United States

Grazing incidence small angle X-ray scattering (GISAXS) methods are frequently very successfully utilized for morphological investigations of thin polymer blend films used in organic photovoltaics. However, conventional GISAXS does no longer provide material sensitivity when the blend consists of more than two components due to the contrast conditions. In case of multi-component blends the use of grazing incidence resonant soft X-ray scattering (GI-RSoXS) can overcome such problems. In this work we exemplarily apply GI-RSoXS to investigate simultaneously the vertical and lateral morphology of polymer-fullerene bulk heterojunction layers made from poly[2,1,3benzothiadiazole-4,7-diyl[4,4-bis(2-ethylhexyl)-4H-cyclopenta[2,1-b:3,4-b']dithiophene2,6-diyl] (PCPDTBT) and [6,6]-penyl-C 61 butyric acid methyl ester (PCBM) with added photosensitizer perylene diimide (PDI) with and without use of the solvent additive 1,8octanedithiol (ODT). The investigation reveals that films without solvent additive ODT tend to show only vertical phase separation while films prepared with solvent additive also phase separate laterally. PDI seems to assist lateral phase separation between PCPDTBT and PCBM by expelling PCBM from the amorphous polymer matrix.

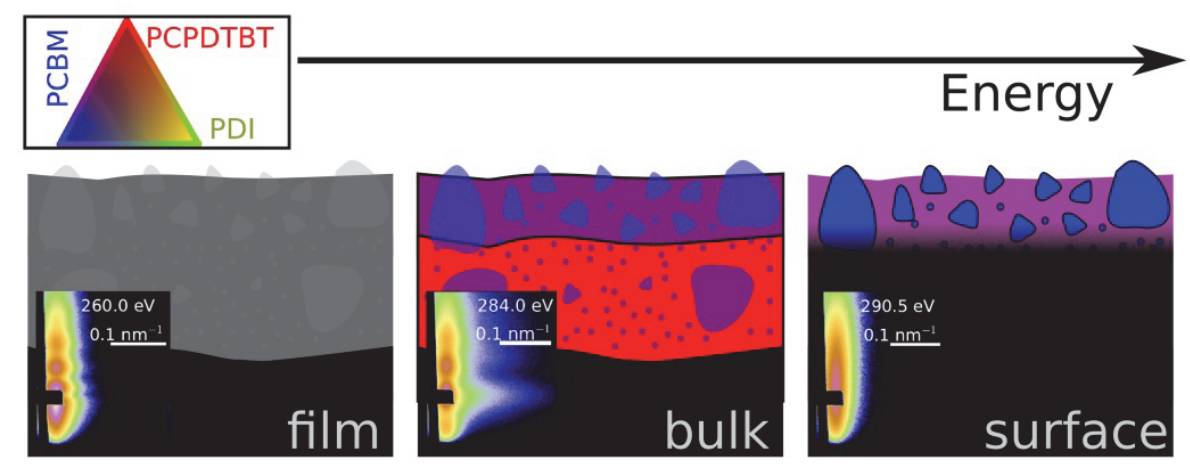

Keywords: GI-RSoXS; GISAXS; polymer blend; conducting polymer; organic photovoltaics 


\section{Introduction}

In most cases the particular functionality of novel polymer materials arises rather from their mesoscale structure than from their intrinsic properties. This holds in particular for polymer composites made from polymer blends. Amongst others, polymer-fullerene blends for use in organic photovoltaic applications have raised strong interest in the last decade[1-5], especially due to their light weight and the potential of a low-cost production using roll-to-roll printing techniques[6-9]. However, for improving both, efficiency and lifetime, it is necessary to fully understand the structure-function relationship, e.g. of the morphology of the blend being the active layer of the organic solar cell and the photovoltaic characteristics[10-15]. Since typical structures are nanometer scaled, their in-depth analysis is demanding due to the limits imposed by the available experimental methods.

Particularly, in the case of thin films of polymer-fullerene blends used for organic photovoltaics, scanning microscopy based methods, such as atomic force microscopy (AFM) or scanning electron microscopy (SEM), are very popular. Both methods are often used to image the topology of thin films on a nanometer scale[15-18] as they provide the fundamental advantage of directly probing the sample surface in the spatial domain. Nevertheless, one must keep in mind that such local methods provide information only on a microscopic sample area. Thus, in order to gain statistically significant information on the nanostructure, a very large number of individual measurements becomes necessary and renders these methods excessively time-consuming. Even more important, such methods cannot provide information on the inner film morphology in a non-destructive way, but only on its surface.

To overcome this lack of bulk-sensitivity, cross-sectional SEM $[15,18]$ is often used which, again, suffers from the low probed film volume and, thus, poor statistics. Furthermore, this method is destructive to the sample and it is not always clear if the blend morphology stays the same when preparing the sample cross section (i.e. breaking of thin film sample). 
When it comes to probing the bulk of a thin film, grazing incidence small angle X-ray scattering (GISAXS) methods have evolved as a reliable probe for nanometer scaled structures and provide a powerful tool[19-23]. Hereby, large sample volumes are typically probed which provide good statistics on the investigated quantities. Moreover, GISAXS methods are non-destructive to the sample. In contrast to microscopy, GISAXS methods provide morphological information only in reciprocal space. As the phase of the scattered waves cannot be recorded, scattering data cannot be translated directly into real space information for directly imaging the blend film morphology. However, prominent length scales and ordered structures are typically derived from the scattering signal by modelling. Particularly for thin film analysis, GISAXS experiments make use of the grazing incidence geometry where the incoming beam impinges the sample under a very shallow angle[20,21,23]. Thus, a large sample area is illuminated. In addition to the most frequently used hard X-rays (about $10 \mathrm{keV}$ ) in GISAXS, also experiments with neutrons, so called grazing incidence small angle neutron scattering (GISANS) measurements have been reported[13,24,25]. Neutron scattering typically demands for rather long exposure times up to hours with the presently available neutron sources. However, by making use of deuteration and contrast matching[26,27], the detected length scales may be asserted to specific sample constituents in complex blend systems. In contrast, hard X-ray scattering experiments do not allow for tuning of the scattering contrast. Therefore, structures may only be detected when the refractive indices of the sample constituents differ sufficiently from each other. Furthermore, no easy information on material-specific substructures can be taken when a blend film consists of more than two constituents. However, as modern synchrotron radiation sources provide highly brilliant X-ray beams, hard X-ray scattering experiments are much faster and can be performed with a high time resolution even in the sub-second domain[2830]. Thus, hard X-ray scattering allows for meaningful in-operando studies that reveal information on the kinetics of thin film formation or degradation[9,12,30-33].

A potential way to include material sensitive scattering and contrast matching to rather fast X-ray scattering experiments arises from the use of soft X-rays[34-41]. While the interaction 
of hard X-rays with matter is mainly described by the local electron density, the interaction with soft X-rays is also strongly dominated by the enhanced electron scattering cross section close to the resonant energies (resonant soft X-ray scattering, RSOXS). As the absorption fine structure of a chemical species is strongly dependent on its chemical environment, the refractive index of different materials can be tuned by changing the X-ray energy.[42] This technique allows for contrast matching and thus enables to differentiate between the different scattering contributions in polymer blend systems with more than two components[43].

Such multi component blends can be of utmost interest for many applications, such as in organic photovoltaics. Besides the initially studied two component blends used for the active layers of organic solar cells, a growing number of studies is investigating three or multicomponent blends [44-49]. Moving from binary to ternary systems is simply increasing the possibilities of tuning the film morphology and thereby improving device characteristics. In one approach the binary polymer-fullerene derivate systems are extended by adding a second polymer to the active layer $[50,51]$ or by adding inorganic materials such as nanoparticles [52] for increasing efficiencies. The use of processing additives, which are typically small molecules, has turned out to be even more successful for boosting device efficiencies $[16,24,53,54]$. In case these processing additives do not evaporate from the initial binary system, ternary systems are created this way. While processing additives should tailor the miscibility and thus the phase separation structure between the major components, small molecules have been added also for other purpose. In this context, for example Hesse et al. have proposed to use photosensitizers to improve the absorption capabilities of the active layer, and consequently the solar cell performance. In more detail, they studied poly[2,1,3benzothiadiazole-4,7-diyl[4,4-bis(2-ethylhexyl)-4H-cyclopenta[2,1-b:3,4-b']dithiophene-2,6diyl]]: [6,6]-penyl-C 61 butyric acid methyl ester (PCPDTBT:PCBM) solar cells and moved to ternary blends by including small amounts of highly absorbing perylene-diimid (PDI) in the PCPDTBT:PCBM blend films[55]. 
In the present study we revisit this ternary system. We report on the application of grazing incidence resonant soft X-ray scattering (GI-RSoXS) on the example of thin films consisting of PCPDTBT:PCBM and PDI, prepared with and without use of $3 \%$ vol. 1,8-octanedithiol (ODT) as solvent additive to chlorobenzene. ODT has formerly been reported to enhance polymer crystallization, the overall phase separation of polymer and fullerene, and, in consequence, to improve the photovoltaic performance[16,36,56,57]. In case ODT residues inside the films a four component system would form. Due to the volatility of ODT in the present study ternary blend films (PCPDTBT:PCBM:PDI) are probed with GI-RSoXS.

\section{Experimental Section}

Sample preparation:

Mother solutions were prepared with a concentration of $30 \mathrm{mg} / \mathrm{ml}(1: 2$ PCPDTBT:PCBM $)$ in chlorobenzene with and without $3 \%$ vol. ODT and split into different samples after materials were fully dissolved (room temperature). The solutions were successively diluted with differently concentrated PDI solutions (or pure solvent, with and without ODT) resulting in sample solutions with concentrations of $\left(20+n^{*} 0.2\right) \mathrm{mg} / \mathrm{ml}$ and compositions of $1: 2: \mathrm{n}^{*} 0.03$ (PCPDTBT:PCBM:PDI) with $n=\{0,1,2,3,4,5,6,10\}$ corresponding to addition of $0,1,2,3,4,5$, 6, 10\% PDI by weight. The materials were used as delivered (PCPDTBT: ONE-material, PCBM: NanoC, PDI, ODT, CB: Sigma Aldrich). The solutions were consecutively cast on silicon $(\mathrm{Si})$ substrates without further treatment via spin coating.

\section{NEXAFS:}

NEXAFS spectra of the pure material films on Si were measured via total electron yield at an angle of $45^{\circ}$ and normalized to the incoming beam intensity for beam energies between 260 and $300 \mathrm{eV}$ in $0.2 \mathrm{eV}$ steps at the soft $\mathrm{X}$-ray scattering beam line 11.0.1.2 at ALS, Berkeley[58]. 


\section{GI-RSoXS:}

GI-RSoXS measurements were performed at the soft X-ray scattering beam line 11.0.1.2 at ALS[58], Berkeley, CA. The incident angle was set to $4^{\circ}$. Sample-to-detector distances of 97.5(2) (films w/o ODT) and 98.3(4) mm (films w/ ODT) were used. The X-rays were polarized (E) perpendicular to the plane of incidence.

\section{Grazing Incidence Resonant Soft X-ray Scattering Measurements on Polymer-}

\section{based Blend Films}

\section{Distinguishing the constituents: NEXAFS and Refractive Indices.}

In resonant soft X-ray scattering (RSoXS) a strong scattering contrast between different sample constituents is obtained due to the strong dependence of their refractive index on their chemical structure. X-ray energies are hereby chosen around the absorption edge of carbon atoms within the species investigated. The chemical environment of these atoms thereby strongly influences the absorption fine structure of the carbon atoms. As a consequence, the refractive index of a specific material becomes a function of energy and strongly differs within different materials. This strong dependence allows for contrast tuning and contrast matching in RSoXS measurements.

However, the refractive index of the investigated materials is typically not known and must, therefore, be determined whenever RSOXS experiments are performed. Unlike the case for hard X-rays, organic materials show strong absorption of X-rays with Energies $E$ in the region around $300 \mathrm{eV}$, providing that the imaginary part $\beta$ of the refractive index $\mathrm{n}$ (see equation 1) must not be neglected.

$$
()=1-()+()
$$


Hereby, $\delta$ denotes the dispersive part of the refractive index. In order to determine the refractive index, both $\delta$ and $\beta$ must be known. Typically, $\delta$ is not directly accessible. However, $\beta$ can be easily determined (at least locally around $300 \mathrm{eV}$ ) by measuring the transmission or total electron yield near edge X-ray absorption fine structure spectra (NEXAFS) of the pure materials. Thereby $\beta$ is determined from the observed mass absorption coefficient $\mu(E)$ (see equation 2), where $\rho$ denotes the mass density, $\mathrm{h}$ and $\mathrm{c}$ are Planck's constant and the speed of light, respectively.

$\delta$ can then be determined from $\beta$ using the Kramers-Kronig relation:

$$
=-20_{\infty}^{\prime}, ' 2-2^{\prime}
$$

. In the present case, NEXAFS spectra were obtained by measuring the total electron yield current which is proportional to the absorbance $[38,59]$. In order to approximate the absorption spectrum for arbitrary energies, the measured absorption spectrum was scaled and extended via the imaginary part of the scattering form factors of all chemical species in the material based on the Henke dataset [60]. Via equation $3 \delta$ is obtained. Densities of 1.1, 1.7 , and $1.5 \mathrm{~g} / \mathrm{cm}^{3}$ were assumed for PCPDTBT, PCBM and PDI based on literature or X-ray reflectivity measurements.

For the given densities, the values of $\delta$ and $\beta$ as well as the critical angle $\alpha_{c}$ and the absorption length $\Lambda=4$ are shown in Figure 1 .

In case of hard X-rays, the optical thicker medium as compared to a polymer is usually vacuum or air. As a consequence, total reflection occurs when the $\mathrm{X}$-ray beam impinges the (polymer) sample under a shallow incident angle below its critical angle from the vacuum or air side. Furthermore, when the X-ray beam impinges under an incident angle that exceeds the critical angle, an evanescent wave appears which couples out under the critical angle with respect to the sample surface. This anomalous surface scattering has been discovered 
by Yoneda and the resulting intensity maximum is called Yoneda peak in literature [61]. In case of hard X-rays, Yoneda scattering has been frequently used for morphological investigations due to its material sensitivity. In this case the critical angle is approximated by $\approx 2$ where $\delta$ is calculated from the chemical composition and the (mostly assumed) mass density via Henke's data set of atomic scattering form factors[60]. Although this approximation holds for hard X-rays where hardly absorption takes place, it breaks down when $\beta$ is no more negligible. It is, however, commonly assumed that Snell's law is valid also when the refractive index becomes complex [62]. Therefore we tentatively assume that the critical angle can be defined as the real part of $\arcsin (n)$ :

$$
=\Re\{\sin -11-+\}
$$



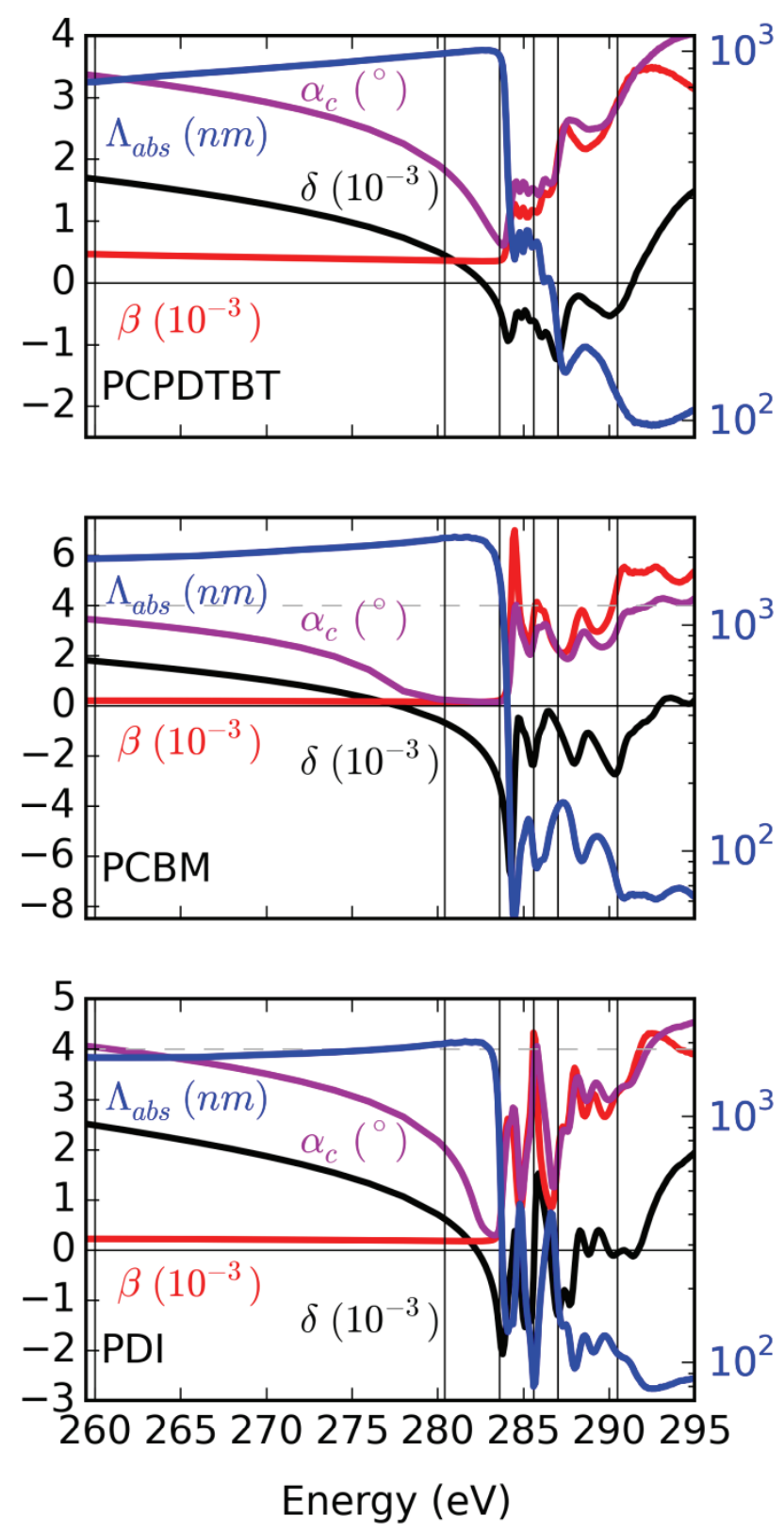

Figure 1. Calculated $\delta$ (black line), $\beta$ (red line) critical angle $\alpha_{\mathrm{c}}$ (purple line), (values on the left axis) and absorption length $\Lambda_{\text {abs }}$ (blue line, in $\mathrm{nm}$, right axis) for the different materials as obtained from NEXAFS measurements. The vertical lines denote specific energies of interest as explained in the text.

When applying equation 5 to the obtained refractive indices, the critical angles shown in Figure 1 are obtained. In comparison, the use of the approximation $\approx 2$ would result in an absence of the real part for some energies, which contradicts the experimentally observed Yoneda scattering. If applicable, the Yoneda peak corresponds to the critical angle where the Fresnel transmission has its maximum. In the present case, s-polarization was chosen for 
the incident waves. For s-polarized waves impinging under the incident angle $\alpha_{i}$ (with respect to the sample surface), the Fresnel transmission coefficient $t$ is given by:

$$
=2 \sin \cos +()()
$$

where $=\sin -1 \cos ()$ is the (complex) transmitted angle (with respect to the sample surface). From the obtained refractive indices we have calculated the Fresnel transmissivities $=2$ as a function of the beam energy $\mathrm{E}$ and the incident angle. Keeping in mind that $\mathrm{T}$ also describes the intensity of an out coupled (evanescent) wave, we can represent the Fresnel transmissivity as a function of $E$ and the vertical momentum loss $q_{z}$ for a fixed incident angle of $4^{\circ}$. This calculation results in the Fresnel transmissivity $T\left(E, q_{z}\right)$ where $q_{z}$ is given by:

$$
(,)=2 \sin +\sin
$$

$\mathrm{T}\left(\mathrm{E}, \mathrm{q}_{z}\right)$ is shown for PCPDTBT, PCBM and PDI in Figure $2 \mathrm{a}$, along with $\mathrm{q}_{z}\left(4^{\circ}, \alpha_{c}\right)$ for the critical angle derived in equation 5. Such calculations were also performed for the different material blends and are given in the supplementary information. As the Yoneda peak also arises at the maximum of the Transmissivity these calculations predict a Yoneda peak around $\mathrm{q}_{\mathrm{z}} \approx 0.17 \mathrm{~nm}^{-1}$ at $260 \mathrm{eV}$. This peak should drop in its $\mathrm{q}_{\mathrm{z}}$-position until it mostly vanishes at around $283 \mathrm{eV}$. In deed such behavior is observed in the measured GI-RSoXS data. Therefore, cuts are taken along the incident plane from the 2D GI-RSoXS data. These cuts are plotted versus the beam energy in Figure $2 \mathrm{~b}$ (upper and center panel). The full set of specular plane cuts versus energy for all samples is provided in the supporting information. Unfortunately, the Yoneda peak is hidden by the beam stop. 


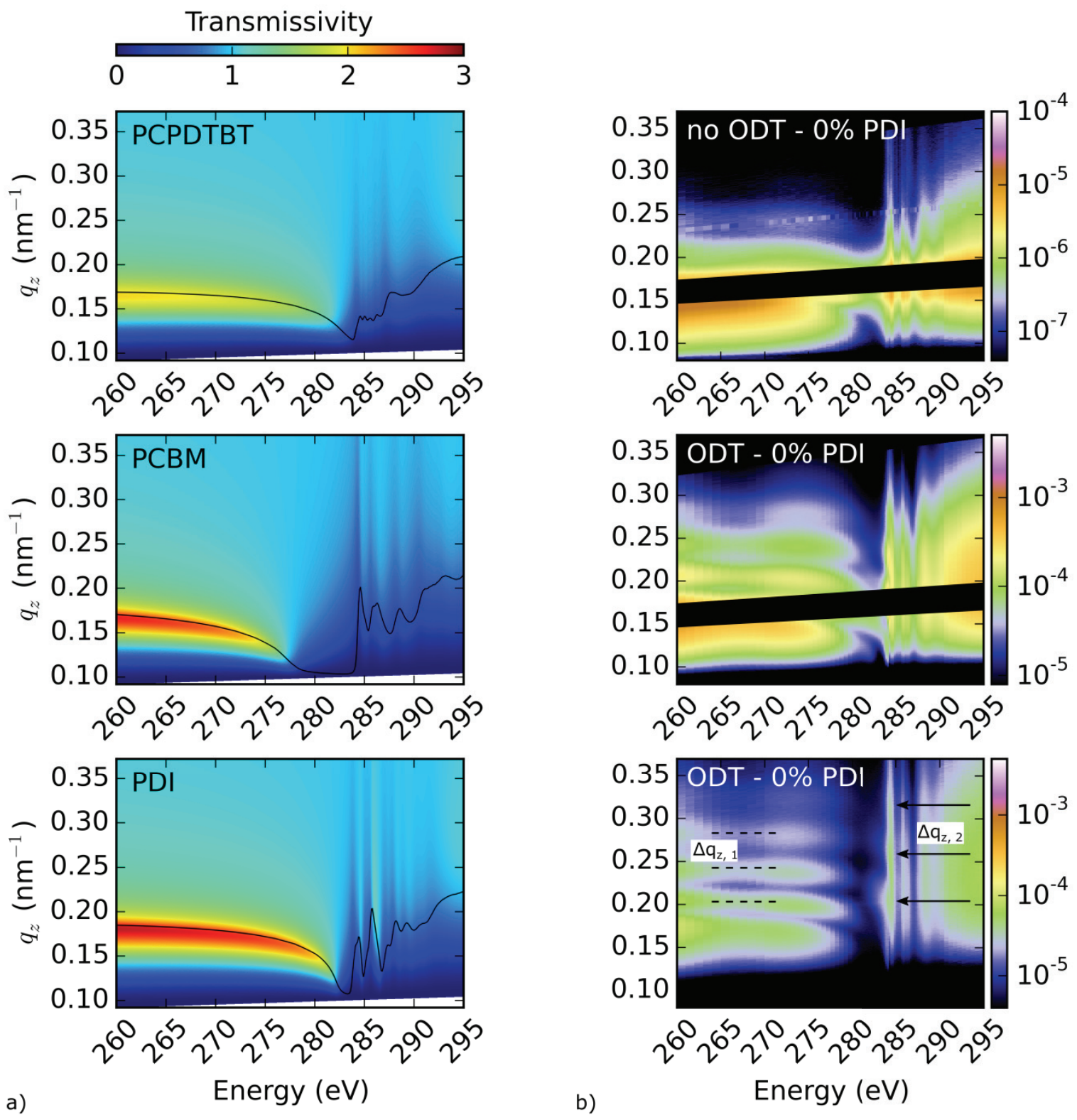

Figure 2. a) Fresnel transmissivities calculated from the refractive indices of PCPDTBT (top), PCBM (middle) and PDI (bottom panel) as obtained from NEXAFS data. The exit angles are transformed to $\mathrm{q}_{z}$ for a fixed incident angle of $4^{\circ}$. The solid lines correspond to $\mathrm{q}_{z}$ of a fixed incident angle of $4^{\circ}$ and a fixed critical angle of $\alpha_{c}$ as obtained via equation 5. b) Exemplary mapping of vertical line cuts of the 2D GI-RSoXS data along the incident plane (top and middle) and with offset (bottom panel) versus X-ray energy for samples w/o PDI. The black stripe denotes the specular beam stop. The pixely stripe in the upper image arises from a few masked, dead pixels of the detector. The dashed lines and the arrows denote the correlated roughness fringes with qz spacings $q_{z, 1}$ and $q_{z, 2}$ correlating to the layer thicknesses $d_{1}$ and $d_{2}$ (see Figure 3).

Thus, a further cut is taken with an offset to the beam stop and shown in the lower panel of Figure $2 b$. A good agreement of the calculated and measured patterns at low $q_{z}$ values is found. The peak arising at around $0.17 \mathrm{~nm}^{-1}$ decreases in q-position (see the lower panel) 
and fades out between 280 and $283 \mathrm{eV}$. As the count statistics is poor for cuts performed off the specular plane in the 2D GI-RSoXS data, this transition is only seen in the centered cuts (upper panels in figure 2b). Since no PDI is present in the measurements shown in Figure $2 \mathrm{~b}$, the nose that arises between 280 and $283 \mathrm{eV}$ is most probably correlated to pure PCPDTBT. This nose appears only when ODT is used, which supports the literature that proposes that ODT leads to enhanced phase separation and thus polymer phases with higher purity.

Interestingly, the calculations predict significantly low intensities from around $284 \mathrm{eV}$ on. Nevertheless, the measured detector cuts show strong scattering amplitudes even above this energy. As bulk scattering is only possible when the incoming beam is transmitted (which is not the case according to the calculations), the high detected intensities must arise from surface scattering, suggesting that from around $284 \mathrm{eV}$ on only surface scattering is found in the GI-RSoXS data. This finding is in good agreement with the 2D GI-RSoXS patterns where no correlated roughness[63] can be observed for energies above $284.4 \mathrm{eV}$, suggesting that the beam does not penetrate the full film any more. Furthermore, this scenario is supported by the sudden drop of absorption length shown in Figure 1.

\section{Correlated Roughness and Buried Interfaces.}

All measured specular cuts from the 2D GI-RSoXS data show a periodically repeating ridge in $q_{z}$ direction. These ridges arise from correlated roughness and are not taken into account for in the calculations. However, these fringes appear when a layer structure is present in which the upper interface of a specific layer conformally follows the topology of its lower interface. Although these fringes do not generically give information on the total film thickness, they can reveal the thickness of the specific layer that shows correlated roughness. In our case, the scattering contrast functions vanish for decreasing energies below $280 \mathrm{eV}$. This means, that by investigating the fringes at energies around $260 \mathrm{eV}$, the total film thickness can be estimated as only low scattering contrasts are expected and 
therefore the film should appear as a homogeneous layer with only one (average) refractive index. By doing so, the film thicknesses $d$ of the different samples can be extracted via:

$\Delta \mathrm{q}_{\mathrm{z}}$ denotes the difference between two intensity maxima of the correlated roughness signal. Interestingly the fringes show a constant (in energy) periodicity for energies below $280 \mathrm{eV}$ and vanish for higher energies. Solely around $284 \mathrm{eV}$ a last set of fringes appears. However, this set of fringes displays a different periodicity. The correlated layer thicknesses are calculated for all PDI contents with and without ODT (see Figure 3).

a)

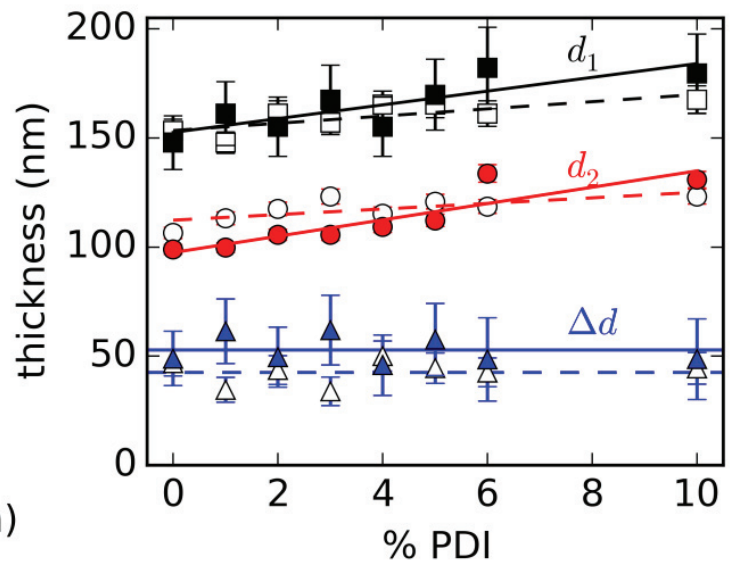

b)

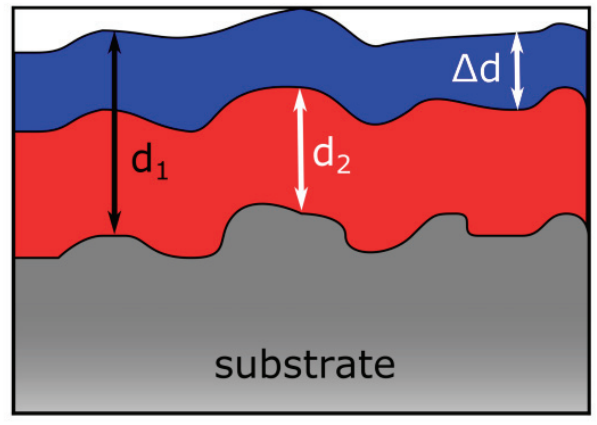

Figure 3. a) Layer thicknesses from correlated roughness at energies $<280 \mathrm{eV}$ (black) and at $284 \mathrm{eV}$ (red). The blue triangles denote the difference of the two layer thicknesses. Open symbols correspond to films processed with ODT, full symbols correspond to films without ODT. The dashed lines are guides to the eye. b) Model of the buried interface with full layer thickness $d_{1}$, lower layer thickness $d_{2}$ and upper layer thickness $\Delta d$. 
The thicknesses estimated from energies $<280 \mathrm{eV}$ suggest film thicknesses growing from around 150 to $180 \mathrm{~nm}$ with increasing PDI content. This growth is reasonable, as PDI was added to the solutions with fixed PCPDTBT:PCBM blend concentrations. Thus, higher amounts of PDI correspond to higher overall concentrations. It is noteworthy that the addition of PDI leads to a stronger growth in thickness when no solvent additive is used, as compared to the case ODT was used for film fabrication. The trend is also visible at $284 \mathrm{eV}$. However, a significantly smaller correlated layer thickness is found there, spanning from around $100 \mathrm{~nm}$ to $120-130 \mathrm{~nm}$ at addition of $10 \%$ PDI. This finding indicates that, whereas the whole film appears homogeneous at below $280 \mathrm{eV}$, a buried interface becomes visible at $284 \mathrm{eV}$. Subtracting the two observed correlated layer thicknesses reveals that their difference remains independent of the PDI content. This observation implies that PDI accumulates in only one of these layers and implies that in all cases, with and without ODT, as well as with and without PDI, vertical phase separation occurs.

\section{Contrast Function and Material Sensitivity.}

The scattered intensity from a structure consisting of two materials $A$ and $B$ is proportional to their scattering contrast function $\Delta n^{2}(4)$.

$$
\Delta 2=\Delta \delta \mathrm{AB} 2+\Delta \quad 2
$$

As $\delta$ and $\beta$ were obtained from the NEXAFS spectrum, $\Delta \mathrm{n}^{2}$ is calculated pairwise for the different material combinations and for the single materials versus vacuum. These scattering contrast functions are depicted in Figure 4. ODT is hereby not accounted for due to its volatility: Most ODT is expected to leave the films soon after preparation. From the contrast functions, several energies of interest are obtained: 


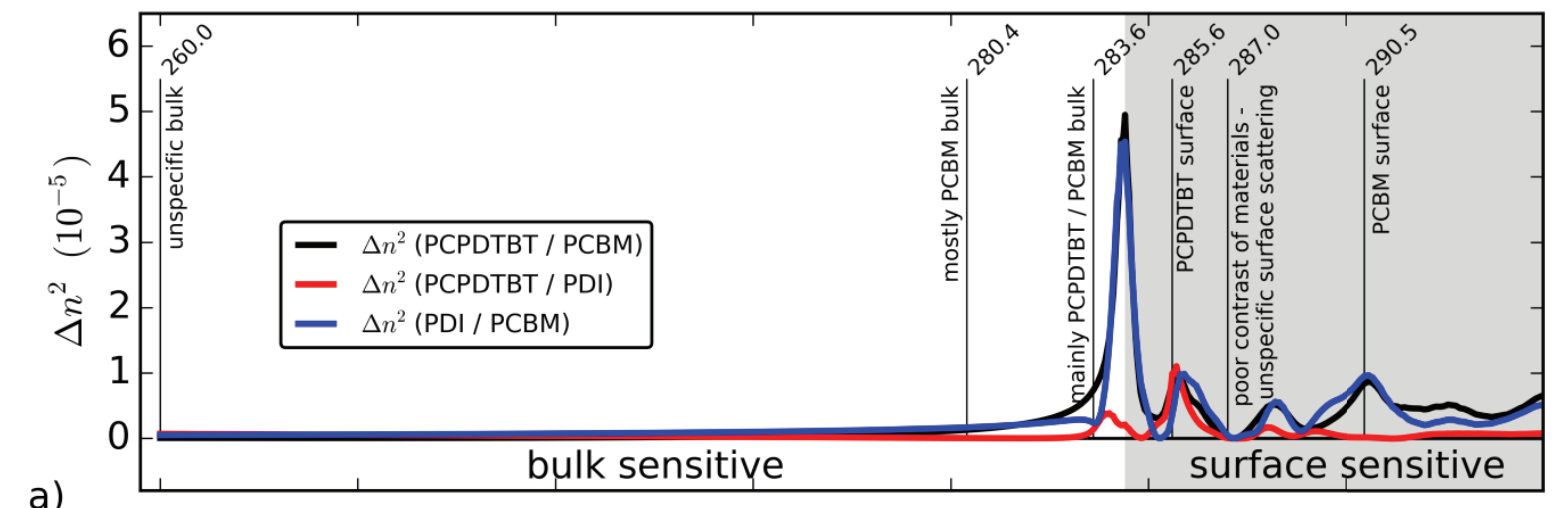

a)

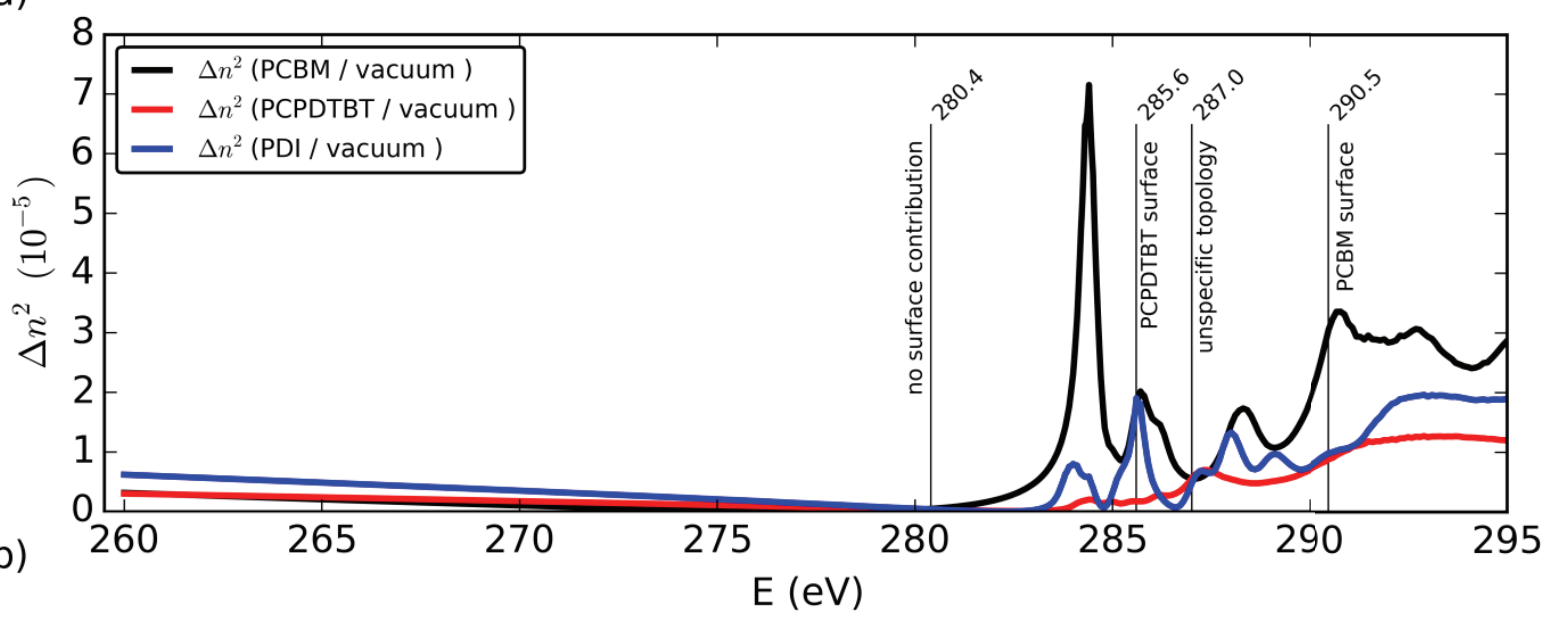

Figure 4. a) Pairwise scattering contrast functions of the different constituents (thick solid lines) as indicated. The different energies of interest are assigned to the particular energies discussed in the main text. b) Contrast functions vs. vacuum as indicated.

\section{Energies of interest:}

- At the lowest investigated energies $(260 \mathrm{eV})$ all three pairwise contrast functions (Figure 4a) are small. Furthermore $\beta$ is negligible. The $X$-ray beam penetrates the full film and no material-specific scattering is expected from any of the constituents.

- At $280.4 \mathrm{eV}$ the contrast functions of PCPDTBT/PCBM and PCBM/PDI exceed the one of PCPDTBT/PDI. Therefore, scattering is expected mostly from PCBM structures. Furthermore, at $280.4 \mathrm{eV}$ the scattering contrast of all three constituents versus vacuum is at its minimum. Therefore, the least surface scattering contribution is expected and we expect the scattering signal to be strongly dominated by bulk scattering. $\beta$ is still negligible and therefore the whole film volume is probed. However, 
the Fresnel transmissivity becomes small around $280 \mathrm{eV}$ and the correlated roughness fringes might fade out due to an overall loss of intensity.

- At around $283.6 \mathrm{eV}$ the contrast function of PCPDTBT/PCBM exceeds the other two. PCPDTBT/PCBM structures are expected to contribute strongest. Slight absorption due to PDI sets on. The film is still fully penetrated and bulk scattering is expected.

- Around $284 \mathrm{eV}$ the beam energy coincides with the carbon resonance in PCBM. Therefore, strongly enhanced scattering contrast is found for PCBM versus anything else. The buried interface described above becomes visible as layers with different PCBM contents show different mean scattering length densities.

- At energies exceeding $284 \mathrm{eV}$, strong absorption hinders the penetration of the full film. Therefore, correlated roughness cannot be observed anymore and the scattering signal is dominated by surface scattering. The contrast functions versus vacuum (Figure $4 b$ ) are of special interest for these energies. Particularly at an energy of $285.6 \mathrm{eV}$, the contrast functions of both, PCBM and PDI, versus air, match, which indicates that structures of PDI or PCBM cannot be distinguished from each other. As PCPDTBT has a different contrast at this energy, pure PCPDTBT structures or voids on the surface will dominate the overall scattering signal.

- At around $287 \mathrm{eV}$ all contrast functions versus air match. Therefore, all materials are indistinguishable and only the surface geometry will cause lateral scattering in GIRSoXS.

- At around $290.5 \mathrm{eV}$ contrast matching of PCPDTBT and PDI versus vacuum occurs. Therefore, $\mathrm{PCBM}$ is the only distinguishable component and lateral scattering is expected to arise mainly from PCBM structures on the surface. 


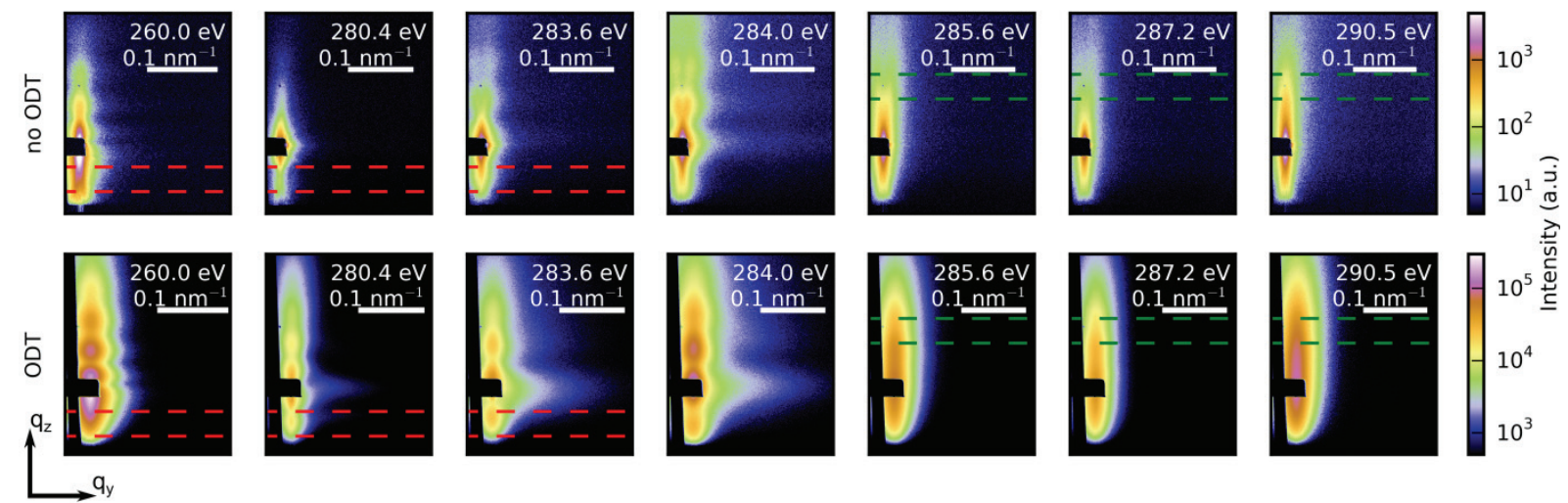

Figure 5. Raw 2D GI-RSoXS patterns of films without PDI for the different X-ray energies of interest as indicated. Data are shown with logarithmic intensity. The scale bar gives an approximation of the $\mathrm{q}_{\mathrm{y}}$ scale and is not valid in $\mathrm{q}_{\mathrm{z}}$-direction. The red and green regions indicate the position of the horizontal line cuts.

Representative 2D GI-RSoXS patterns for the named energies are shown in Figure $\mathbf{5}$ for the cases with and without ODT but without PDI. A full set including all PDI contents is provided in the supplemental information. Anyhow, in a qualitative analysis the PDI content does not significantly change the 2D GI-RSoXS patterns. Therefore, the addition of PDI is expected to induce only small changes which are discussed in a framework of data modeling. In order to understand the main scattering features, however, we first draw detail on the scattering patterns without PDI in the context of the theoretical discussion given above. For investigating the bulk morphology, the focus is put on the scattering data at $260,280.4$, and $283.6 \mathrm{eV}$ :

- At $260 \mathrm{eV}$, correlated roughness is observed with and without use of ODT. At this energy, both PCPDTBT and PCBM have a similar contrast versus vacuum. Thus, the impinging X-ray interacts with a homogeneous film surface which mimics the substrate interface roughness and therefore causes the observed fringes.

- At $\mathbf{2 8 0 . 4} \mathrm{eV}$ mostly bulk PCBM scattering is expected. Correlated roughness fringes should appear but are hardly visible in the scattering patterns due to the overall low diffuse scattering signal for energies around $280 \mathrm{eV}$. Since the film consists mostly of PCBM which displays a very low Fresnel transmissivity in this energy region (see 
Figure 2a) and since surface scattering is negligible due to the vanishing contrast of the individual constituents versus vacuum (see Figure 4b), the overall diffuse scattering signal is very low around $280 \mathrm{eV}$.

- At $283.6 \mathrm{eV}$ PCPDTBT/PCBM scattering is enhanced. In case no ODT is used, we observe a rod-like split scattering pattern in the high $\mathrm{q}_{\mathrm{z}}$ region which suggests ordered domain spacing. The split signal appears both, at higher energies where only the surface is probed and in the low $q_{z}$ region at lower energies. Therefore, we suggest that these structures appear both on the surface and in the bulk film. Such structures are not observed in the films prepared with ODT.

Between 284.2 and $284.4 \mathrm{eV}$ (not shown) the fringes disappear and all scattering images from higher energies look similar to the ones shown in Figure 5. This loss of fringes in the GIRSoXS data indicates that the X-rays do not penetrate the full sample any more. Thus, at 285.6, 287, and $290.5 \mathrm{eV}$ all patterns become similar and allow probing only the film surface: In order to understand the influence of solvent additive and PDI on the bulk and surface morphology, horizontal line cuts from the 2D GI-RSoXS data were taken a) at the Yoneda region (marked red in Figure 5) for bulk sensitive energies (260, 280.4, and 283.6 eV) and above the beam stop for surface-sensitive energies (285.6, 287, and $290.5 \mathrm{eV}$, marked green in Figure 5). Between 260 and $283.6 \mathrm{eV}$ a fixed Integration area was chosen where the Yoneda peak is always included. The data were fitted in the framework of the effective interface approximation (EIA) and local monodisperse approximation (LMA) using a model with three different sub structures $[19,64]$. Each sub structure is hereby characterized by a most-probable domain radius, a most probable inter distance of domains in a 1-dimensional paracrystal after Hosemann et al. [65], and an independent intensity that relates to the occurrence of each specific sub structure. The corresponding model curves using EIA and LMA are shown with the horizontal line cuts in Figure 6. The corresponding model parameters are depicted in Figure 7. 


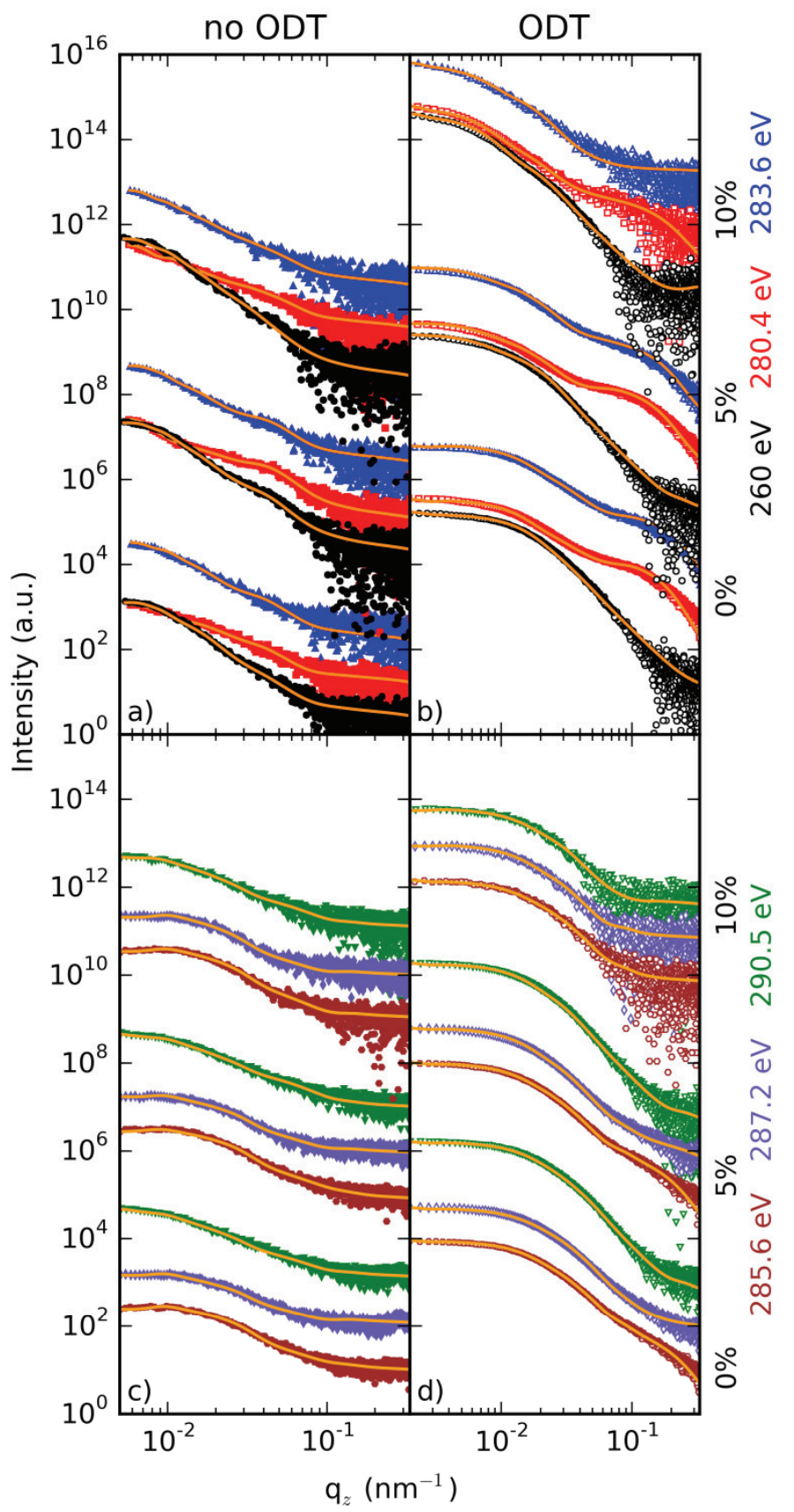

Figure 6. Horizontal line cuts of the 2D GI-RSoXS data at different energies of samples processed without ( $a$ and $c$ ) and with ODT ( $b$ and $d$ ). $a+b)$ Selected bulk-sensitive energies for which the cuts were taken around the Yoneda peak position for energies around $283 \mathrm{eV}$ (Indicated red in Figure 5). $c+d$ ) Selected surface-sensitive energies for which the cuts were extracted at high $\mathrm{q}_{\mathrm{z}}$ values (indicated green in Figure 5). The orange lines represent the fit model curves for extracting quantitative information. All curves were grouped and shifted for clarity. The energies are coded by colors (within group from bottom to top): $a+b): 260 \mathrm{eV}$ (black), $280.4 \mathrm{eV}$ (red), $283.6 \mathrm{eV}$ (blue), c+d): $285.6 \mathrm{eV}$ (brown), $287.2 \mathrm{eV}$ (purple), $290.5 \mathrm{eV}$ (green). 
no ODT

bulk
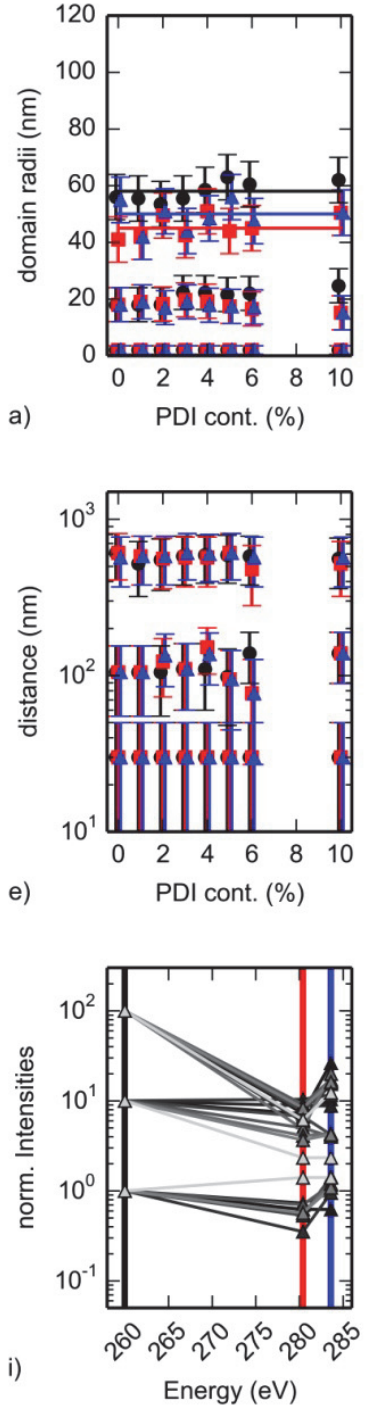

surface

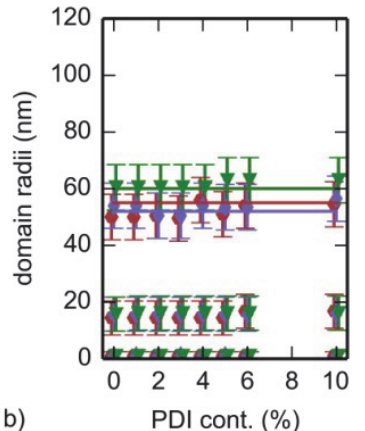

b)
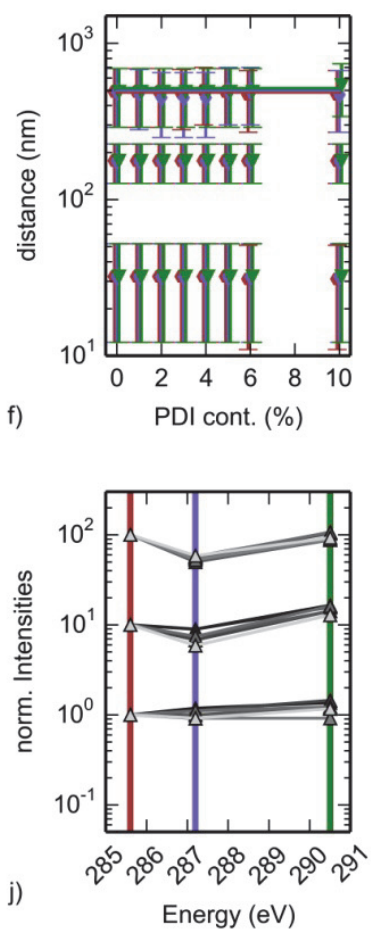

ODT

bulk
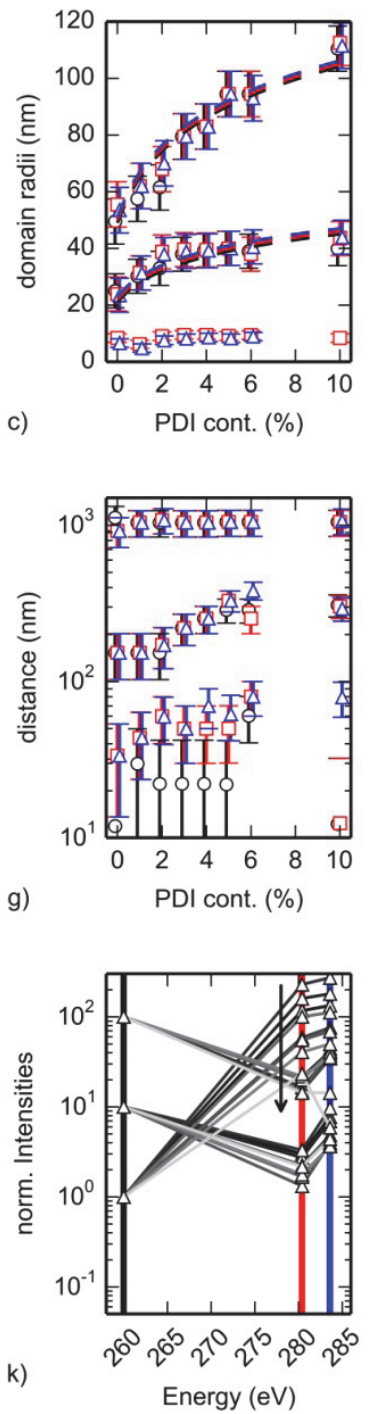

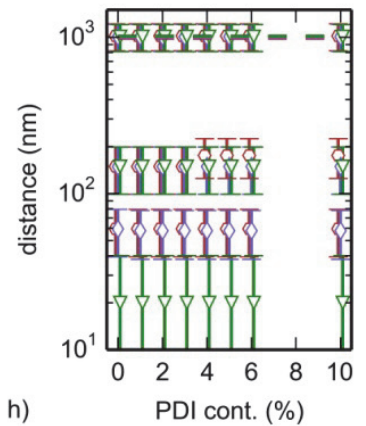

surface
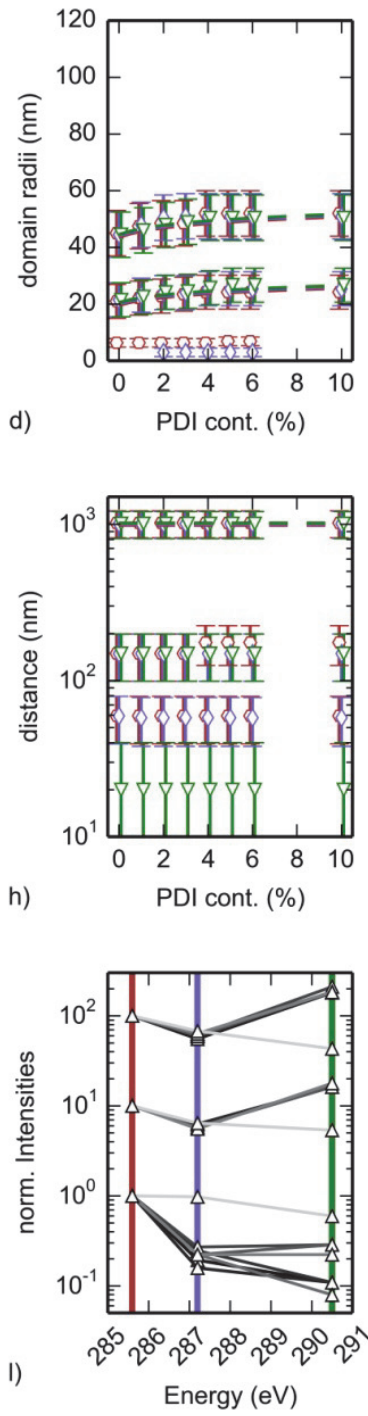

Figure 7. Fitting results from GI-RSoXS data. a,c,e,g) Fitting results for line cuts at the Yoneda peak position for bulk sensitive energies (black: $260 \mathrm{eV}$, red: $280.4 \mathrm{eV}$, and blue: $283.6 \mathrm{eV})$. b,d,f,h) Fitting results for line cuts at a higher $q_{z}$ value $\left(0.3 \mathrm{~nm}^{-1}\right)$ for the different surface sensitive energies (brown: $258.6 \mathrm{eV}$, purple: $287 \mathrm{eV}$, and green: $290.5 \mathrm{eV}$ ) The solid and dashed lines in a-h) are guides to the eye for films without and with ODT, respectively. Full symbols correspond to films without ODT, open symbols to films prepared with ODT. i-I) show the intensities of the scattering contributions of the different small, intermediate and large sub structures (normalized by their values at $260 \mathrm{eV}$ and $285.4 \mathrm{ev}$, respectively.). The grey values of the lines encode the PDI content where black refers to $0 \%$ PDI and the brightest grey refers to $10 \%$ PDI. The arrow in k) denotes the loss of scattering intensity of the small domains with growing PDI content in case ODT is used. Intensity evolutions groupwise shifted for clarity in $\mathrm{i}-\mathrm{I})$. The colored vertical lines denote the energies and their color code used in panels a-h). 
A first difference between films processed with and without ODT is clearly manifested in the domain sizes (Figure 7a and 7c) and inter distances (Figure 7e and 7g) inside the bulk of the film. For all concentrations and length scales, domains are larger and further apart from each other when ODT is used as compared to the case without ODT. This behavior is well known from literature on the influence of solvent additives on PCPDTBT:fullerene blends and resembles solvent-additive induced phase separation on a nanometer scale $[36,56,57]$. Figure $7 \mathrm{c}$ and $7 \mathrm{~g}$ further suggest that the film morphology of films processed with ODT is strongly dependent on the amount of PDI added. When ODT is used, growth of the large and intermediate (length scale of 40 to $120 \mathrm{~nm}$ radius) domains with increasing PDI content is observed for all probed energies both on the surface and in the bulk. However, stronger growth is found thereby in the bulk film. Figures $7 \mathrm{~g}$ and $7 \mathrm{~h}$ show the evolution of inter distances for films prepared with ODT. Interestingly, growth is observed only for intermediate and smaller structures in the bulk film whereas the inter distances stay independent of the PDI content in the surface of the film. Unlike, both the distances and sizes seem to be fully unaffected by PDI when no ODT is used, which makes sense in a well intermixed film (Figures $7 \mathrm{e}$ and $7 \mathrm{f}$ ). Moreover, as these quantities identical in the surface and the bulk, we propose that they are mainly located in the surface. The domain growth with ODT displays in a shift of the line cut intensities to lower $q_{y}$-values in the region of $q_{y}<0.02 \mathrm{~nm}^{-2}$ in Figure $6 \mathrm{~b}$ and $6 \mathrm{~d}$ and is observed both in the bulk-sensitive and the surface-sensitive regime. However, larger domains are thereby generally found for bulk sensitive measurements than for the surface sensitive ones. These findings suggest that PDI can further enhance phase separation in PCPDTBT:PCBM films processed with ODT. In this case large PCBM domains that form close to the interface are, furthermore, found to broaden towards the bulk phase like an iceberg on the macroscopic scale. This is compatible with the growing inter distances of smaller and intermediate domains as these will vanish in favor of larger ones. Therefore, we suggest an Ostwald-ripening like process. Without ODT a fully different behavior is observed. In case no ODT is used, the film morphology is found fully independent of the amount of PDI added. However, slightly smaller domains are found on the surface than in the 
bulk, suggesting that aggregation can only take place close to the surface. This observation is also supported by the lack of small aggregates. In case ODT is used, small aggregates are found with radii on the length scale of 5 to $10 \mathrm{~nm}$ mostly in the bulk. These small aggregates in the bulk are particularly indicated by the shoulder in the scattering signal of the films with ODT (Figure 6b). At energies where PCBM shows increasingly strong interaction (280.4, and $283.6 \mathrm{eV})$, a prominent shoulder in the line cuts at $\mathrm{q}_{\mathrm{y}}>0.1 \mathrm{~nm}^{-1}$ appears. Interestingly, this shoulder is less pronounced when large amounts of PDI are used (10\%). Since these peaks arise for energies close to the PCBM resonance edge, we suggest that these domains consist of PCBM. It is noteworthy that these small domains have formerly been identified as a reason for improving solar cell performance when ODT is used. Data modeling, however, suggests that the size of these small aggregates is not influenced by adding PDI. However, we find that, while leading to large aggregate formation, increasing amounts of PDI continuously suppresses the formation of these small aggregates. This finding cannot be extracted from the inter distances since these cannot be extracted with sufficient precision. However, the conclusion can be made from analyzing the evolution of sub structure scattering intensities with different energies (Figure 7i-7I). In the corresponding graphs, the intensities are normalized to their values at 260 and $285.6 \mathrm{eV}$ for the bulk and surface specific energy ranges, respectively. Most sub structure scattering intensities drop from 260 to $280.4 \mathrm{eV}$ due to the loss in Fresnel transmissivity and then recover towards $283.6 \mathrm{eV}$ due to the proximity to the PCBM carbon resonance. A similar behavior is found for the surfacesensitive energies where the minimum scattering intensity is found at $287 \mathrm{eV}$. At this energy contrast matching and a lowest scattering contrast versus vacuum for all materials and material combinations are found. Solely, the small sub structure in the bulk films prepared with ODT shows a significantly different behavior (Figure 7k). Instead of losing intensity, the sub structure scattering intensity increases by partially more than two decades from 260 to 280.4 eV and further increases for low PDI contents towards $283.6 \mathrm{eV}$. As these energies lead to strong PCBM scattering we suggest that the corresponding sub structure consists of small PCBM domains that are only present in bulk films with ODT. Furthermore, for the 
surface sensitive region, higher scattering intensities are found for all sub structures (except for the small ones when ODT is used) at $290.5 \mathrm{eV}$ as compared to 285.6 and $287.2 \mathrm{eV}$. As for $290.5 \mathrm{eV}$ contrast matching of PDI and PCPDTBT occurs, this indicates that the surface structures are, indeed, PCBM agglomerates.

\section{Discussion: The Model of Film Morphology}

Using GI-RSoXS we are able to find several dependencies of the morphology on the presence of ODT and PDI. Based on these observations we suggest the following model of the morphology of the different PCPDTBT:PCBM blend films prepared with and without ODT as solvent additive and PDI as photo sensitizer for PCBM. A graphical representation of this model is shown in Figure 8.

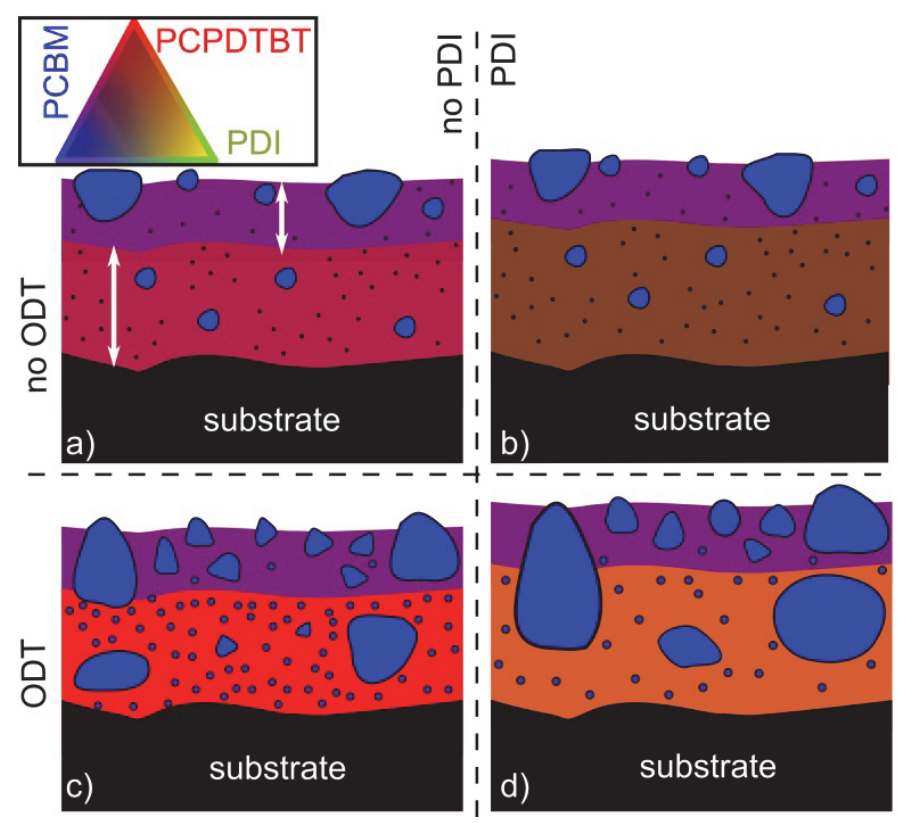

Figure 8. Graphical representation of PCPDTBT:PCBM:PDI thin films. $a+b)$ When no ODT is used, the components mix relatively well and PDI can be included in the overall amorphous blend. However, vertical phase separation into a polymer- and fullerene-rich layer occurs. PCBM aggregation is mainly present at the surface of the fullerene-rich top layer. PDI is mainly located in the polymer-rich layer which grows in thickness when PDI is added. $c+d$ ) When ODT is used, lateral phase separation occurs, additionally to the vertical phase separation. PDI repels PCBM from the amorphous polymer matrix. Therefore PDI is located mainly in the lower polymer-rich phase. At higher PDI concentrations, most PCBM is expelled 
from the polymer matrix and no more small PCBM domains can form in the polymer-rich phase.

The appearance of correlated roughness fringes at different energies with different periodicities lead to the conclusion that all films have a buried interface, even when no PDI is present. Thus, a fullerene-rich and a polymer-rich layer must form and vertically phase separate. The thicker of these layers (approximately $100 \mathrm{~nm}$ ) solely grows with growing amounts of PDI added. Thus, PDI must migrate to only one of these phases. Lateral scattering leads to the conclusion, that without ODT, PCBM agglomerates form only at the surface. This induces that the surface layer is the fullerene-rich layer as precipitations only form when the solubility limit of PCBM in the polymer matrix is exceeded. Therefore, PDI must rather embed in the lower, polymer-rich layer and cause a growing lower layer thickness with increasing PDI concentrations. Concerning the suppression of small PCBM aggregates, we propose that PDI molecules interact stronger with the amorphous polymer phase than PCBM. In this case, PDI will repel PCBM molecules from the polymer matrix. If this happens already during thin film formation, an increased amount of PCBM will reach the PCBM layer and therefore, larger PCBM aggregates will form. At the same time, less PCBM is available in the polymer-rich layer. Therefore, a smaller amount of small PCBM agglomerates can form.

The latter mechanism might be of particular interest when it comes to transferring our observations to photovoltaic devices. Hesse et al. have proposed the use of PDI as a photo sensitizer for PCBM in organic photovoltaic devices. Thereby it was used to enhance light absorption in the visible region. While this concept was found to work well in bilayer devices, it failed in PCPDTBT:PCBM:PDI bulk heterojunction devices: Whereas addition of up to $2 \%$ PDI has resulted in an increased performance, any higher concentration was disadvantageous. Hesse et al. suggested that morphological limitations caused the loss of performance at higher concentrations. Based on our model we suggest that the suppression 
of small PCBM domains in the bulk layer will lead to a loss of both, interfacial area and percolation, and therefore deteriorate the overall photovoltaic performance.

\section{Conclusions}

In the present study we have presented grazing incidence resonant soft X-ray scattering as an advanced scattering method for morphological investigations on a nanometer scale of thin organic blend films. We could show that GI-RSoXS is suitable for investigating simultaneously both, the lateral and the vertical film morphology, which is usually not easily possible in conventional hard X-ray scattering methods. The strong dependence of refractive indices of organic materials in the energy range from 260 to $300 \mathrm{eV}$ allows for conclusions on the material composition of nanometer scaled structures on the investigated films. All these possibilities render GI-RSoXS as an extremely powerful tool for nanoscale morphology investigations on thin films.

We successfully use GI-RSoXS to decrypt the morphologies of PCPDTBT:PCBM based thin films with different contents of PDI as photo sensitizer for organic photovoltaics. The role of solvent additive ODT as driving agent for phase separation could be verified. The data suggest vertical phase separation into a fullerene-rich top and a polymer-rich bottom layer for all probed PDI contents, irrespective of the presence of ODT. Furthermore, we could clarify the role of PDI on the morphology of these films. Thereby, PDI turns out to further assist solvent-additive driven phase separation, whereas it does not induce phase separation by itself. The results nicely explain why the concept of PDI as photosensitizer for the electron acceptor in OPV, which was formerly found to work in bilayer cells, fails in the bulkheterojunction devices due to morphological limitations. 


\section{Acknowledgments}

Financial support is acknowledged by TUM.solar in the frame of the Bavarian Collaborative Research Project "Solar technologies go Hybrid" (SolTec), by the GreenTech Initiative (Interface Science for Photovoltaics - ISPV) of the EuroTech Universities and by the Nanosystems Initiative Munich (NIM). A.H. acknowledges funding from the Early Career DOE program and C.J.S. from the Bavarian State Ministry of Education, Science and the Arts via the International Graduate School "Materials Science of Complex Interfaces" (Complnt). The Advanced Light Source is supported by the Director, Office of Science, Office of Basic Energy Sciences, of the U.S. Department of Energy under Contract No. DE-AC02$05 \mathrm{CH} 11231$.

\section{References}

[1] C.J. Brabec, S. Gowrisanker, J.J.M. Halls, D. Laird, S. Jia, S.P. Williams, Adv. Mater. 22 (2010) 3839-3856.

[2] G. Dennler, M.C. Scharber, C.J. Brabec, Adv. Mater. 21 (2009) 1323-1338.

[3] Y. Liang, Z. Xu, J. Xia, S.-T. Tsai, Y. Wu, G. Li, C. Ray, L. Yu, Adv. Mater. 22 (2010) E135-E138.

[4] Z. He, B. Xiao, F. Liu, H. Wu, Y. Yang, S. Xiao, C. Wang, T.P. Russell, Y. Cao, Nat. Photonics 9 (2015) 174-179.

[5] G. Yu, J. Gao, J.C. Hummelen, F. Wudl, A.J. Heeger, Science 270 (1995) 17891791.

[6] T. Agostinelli, T.A.M. Ferenczi, E. Pires, S. Foster, A. Maurano, C. Müller, A.

Ballantyne, M. Hampton, S. Lilliu, M. Campoy-Quiles, H. Azimi, M. Morana, D.D.C. Bradley, J. Durrant, J.E. Macdonald, N. Stingelin, J. Nelson, J. Polym. Sci. Part B Polym. Phys. 49 (2011) 717-724.

[7] F.C. Krebs, T. Tromholt, M. Jørgensen, Nanoscale 2 (2010) 873.

[8] R. Søndergaard, M. Hösel, D. Angmo, T.T. Larsen-Olsen, F.C. Krebs, Mater. Today 15 (2012) 36-49.

[9] S. Pröller, F. Liu, C. Zhu, C. Wang, T.P. Russell, A. Hexemer, P. Müller-Buschbaum, E.M. Herzig, Adv. Energy Mater. (2015) 1501580.

[10] S. Bertho, G. Janssen, T.J. Cleij, B. Conings, W. Moons, A. Gadisa, J. D’Haen, E. Goovaerts, L. Lutsen, J. Manca, D. Vanderzande, Sol. Energy Mater. Sol. Cells 92 (2008) 753-760.

[11] B. Conings, S. Bertho, K. Vandewal, A. Senes, J. Dhaen, J. Manca, R.A.J. Janssen, Appl. Phys. Lett. 96 (2010) 163301-163301-3. 
[12] C.J. Schaffer, C.M. Palumbiny, M.A. Niedermeier, C. Jendrzejewski, G. Santoro, S.V. Roth, P. Müller-Buschbaum, Adv. Mater. 25 (2013) 6760-6764.

[13] M.A. Ruderer, R. Meier, L. Porcar, R. Cubitt, P. Müller-Buschbaum, J. Phys. Chem. Lett. 3 (2012) 683-688.

[14] D. Chirvase, J. Parisi, J.C. Hummelen, V. Dyakonov, Nanotechnology 15 (2004) 1317.

[15] H. Hoppe, N.S. Sariciftci, J. Mater. Chem. 16 (2006) 45-61.

[16] J. Peet, J.Y. Kim, N.E. Coates, W.L. Ma, D. Moses, A.J. Heeger, G.C. Bazan, Nat. Mater. 6 (2007) 497-500.

[17] S.H. Park, A. Roy, S. Beaupre, S. Cho, N. Coates, J.S. Moon, D. Moses, M. Leclerc, K. Lee, A.J. Heeger, Nat Photon 3 (2009) 297-302.

[18] H. Hoppe, M. Niggemann, C. Winder, J. Kraut, R. Hiesgen, A. Hinsch, D. Meissner, N.S. Sariciftci, Adv. Funct. Mater. 14 (2004) 1005-1011.

[19] R. Lazzari, J. Appl. Crystallogr. 35 (2002) 406-421.

[20] D. Smilgies, P. Busch, C.M. Papadakis, D. Posselt, Synchrotron Radiat. News 15 (2002) 35-42.

[21] A. Hexemer, P. Müller-Buschbaum, IUCrJ 2 (2015) 106-125.

[22] P. Müller-Buschbaum, Adv. Mater. (2014) 7692-7709.

[23] P. Müller-Buschbaum, Anal. Bioanal. Chem. 376 (2003) 3-10.

[24] S. Guo, B. Cao, W. Wang, J.-F. Moulin, P. Müller-Buschbaum, ACS Appl. Mater. Interfaces 7 (2015) 4641-4649.

[25] P. Müller-Buschbaum, Polym. J. 45 (2013) 34-42.

[26] P. Auroy, L. Auvray, L. Leger, Macromolecules 24 (1991) 2523-2528.

[27] M.M. Antxustegi, P.J. Hall, J.M. Calo, J. Colloid Interface Sci. 202 (1998) 490-498.

[28] K. Sarkar, E.V. Braden, S. Pogorzalek, S. Yu, S.V. Roth, P. Müller-Buschbaum, ChemSusChem 7 (2014) 2140-2145.

[29] M. Schwartzkopf, G. Santoro, C.J. Brett, A. Rothkirch, O. Polonskyi, A. Hinz, E. Metwalli, Y. Yao, T. Strunskus, F. Faupel, P. Müller-Buschbaum, S.V. Roth, ACS Appl. Mater. Interfaces 7 (2015) 13547-13556.

[30] K.W. Chou, B. Yan, R. Li, E.Q. Li, K. Zhao, D.H. Anjum, S. Alvarez, R. Gassaway, A. Biocca, S.T. Thoroddsen, A. Hexemer, A. Amassian, Adv. Mater. 25 (2013) 1923-1929.

[31] C.M. Palumbiny, F. Liu, T.P. Russell, A. Hexemer, C. Wang, P. Müller-Buschbaum, Adv. Mater. 27 (2015) 3341-3341.

[32] W. Wang, C.J. Schaffer, L. Song, V. Körstgens, S. Pröller, E.D. Indari, T. Wang, A. Abdelsamie, S. Bernstorff, P. Müller-Buschbaum, J. Mater. Chem. A 3 (2015) 8324-8331. 
[33] J.T. Rogers, K. Schmidt, M.F. Toney, G.C. Bazan, E.J. Kramer, J. Am. Chem. Soc. 134 (2012) 2884-2887.

[34] H. Ade, A.P. Hitchcock, Polymer 49 (2008) 643-675.

[35] S. Swaraj, C. Wang, T. Araki, G. Mitchell, L. Liu, S. Gaynor, B. Deshmukh, H. Yan, C.R. McNeill, H. Ade, Eur. Phys. J. Spec. Top. 167 (2009) 121-126.

[36] Y. Gu, C. Wang, T.P. Russell, Adv. Energy Mater. 2 (2012) 683-690.

[37] C.R. McNeill, N.C. Greenham, Adv. Mater. 21 (2009) 3840-3850.

[38] B.A. Collins, J.E. Cochran, H. Yan, E. Gann, C. Hub, R. Fink, C. Wang, T. Schuettfort, C.R. McNeill, M.L. Chabinyc, H. Ade, Nat. Mater. 11 (2012) 536-543.

[39] S.C.B. Mannsfeld, Nat. Mater. 11 (2012) 489-490.

[40] J. Wernecke, H. Okuda, H. Ogawa, F. Siewert, M. Krumrey, Macromolecules 47 (2014) 5719-5727.

[41] M.A. Ruderer, C. Wang, E. Schaible, A. Hexemer, T. Xu, P. Müller-Buschbaum, Macromolecules 46 (2013) 4491-4501.

[42] J. Stöhr, NEXAFS Spectroscopy, Springer Science \& Business Media, 2013.

[43] C. Wang, D.H. Lee, A. Hexemer, M.I. Kim, W. Zhao, H. Hasegawa, H. Ade, T.P. Russell, Nano Lett. 11 (2011) 3906-3911.

[44] T. Ameri, P. Khoram, J. Min, C.J. Brabec, Adv. Mater. 25 (2013) 4245-4266.

[45] L. Lu, T. Xu, W. Chen, E.S. Landry, L. Yu, Nat. Photonics 8 (2014) 716-722.

[46] P.P. Khlyabich, B. Burkhart, B.C. Thompson, J. Am. Chem. Soc. 133 (2011) 1453414537.

[47] L. Ye, S. Zhang, W. Ma, B. Fan, X. Guo, Y. Huang, H. Ade, J. Hou, Adv. Mater. 24 (2012) 6335-6341.

[48] M. Campoy-Quiles, Y. Kanai, A. El-Basaty, H. Sakai, H. Murata, Org. Electron. 10 (2009) 1120-1132.

[49] R. Lin, M. Wright, B. Puthen Veettil, A. Uddin, Synth. Met. 192 (2014) 113-118.

[50] H. Mangold, A.A. Bakulin, I.A. Howard, C. Kästner, D.A.M. Egbe, H. Hoppe, F. Laquai, Phys. Chem. Chem. Phys. 16 (2014) 20329-20337.

[51] V. Vohra, M. Campoy-Quiles, M. Garriga, H. Murata, J. Mater. Chem. 22 (2012) 20017-20025.

[52] D.M. González, V. Körstgens, Y. Yao, L. Song, G. Santoro, S.V. Roth, P. MüllerBuschbaum, Adv. Energy Mater. 5 (2015) 1401770.

[53] L. Lu, L. Yu, Adv. Mater. 26 (2014) 4413-4430. 
[54] D. Huang, Y. Li, Z. Xu, S. Zhao, L. Zhao, J. Zhao, Phys. Chem. Chem. Phys. 17 (2015) 8053-8060.

[55] H.C. Hesse, J. Weickert, C. Hundschell, X. Feng, K. Müllen, B. Nickel, A.J. Mozer, L. Schmidt-Mende, Adv. Energy Mater. 1 (2011) 861-869.

[56] C.J. Schaffer, J. Schlipf, E. Dwi Indari, B. Su, S. Bernstorff, P. Muller-Buschbaum, ACS Appl. Mater. Interfaces (2015).

[57] H.-C. Liao, C.-S. Tsao, Y.-T. Shao, S.-Y. Chang, Y.-C. Huang, C.-M. Chuang, T.-H. Lin, C.-Y. Chen, C.-J. Su, U.-S. Jeng, Y.-F. Chen, W.-F. Su, Energy Environ. Sci. 6 (2013) 1938-1948.

[58] C. Wang, A. Hexemer, J. Nasiatka, E.R. Chan, A.T. Young, H.A. Padmore, W.F. Schlotter, J. Lüning, S. Swaraj, B. Watts, E Gann, H. Yan, H. Ade, IOP Conf. Ser. Mater. Sci. Eng. 14 (2010) 012016.

[59] B. Watts, S. Swaraj, D. Nordlund, J. Lüning, H. Ade, J. Chem. Phys. 134 (2011) 024702.

[60] B.L. Henke, J.C. Davis, E.M. Gullikson, R.C.C. Perera, Lawrence Berkeley Natl. Lab. (1988).

[61] Y. Yoneda, Phys. Rev. 131 (1963) 2010-2013.

[62] Max Born \& Emil Wolf, Principles of Optics (4th.ed.) Pergamon Press 1970

[63] P. Müller-Buschbaum, M. Stamm, Macromolecules 31 (1998) 3686-3692.

[64] G. Renaud, R. Lazzari, F. Leroy, Surf. Sci. Rep. 64 (2009) 255-380.

[65] R. Hosemann, W. Vogel, D. Weick, F.J. Baltá-Calleja, Acta Crystallogr. Sect. A 37 (1981) 85-91. 